\title{
Visual working memory can selectively reset a subset of its representations
}

\author{
Halely Balaban $^{1,2} \cdot$ Trafton Drew $^{3} \cdot$ Roy Luria ${ }^{1,2}$
}

Published online: 9 November 2017

(C) Psychonomic Society, Inc. 2017

\begin{abstract}
The visual working memory (VWM) resetting process is triggered when the mapping between an object in the environment and its corresponding VWM representation becomes irrelevant. Resetting involves discarding the no longer relevant representations, and encoding novel representations and mappings. We examined how resetting operates on VWM's contents. Specifically, we tested whether losing only part of the encoded mappings led to resetting all of the VWM representations. Subjects monitored moving polygons for an abrupt shape-change. Occasionally, a polygon separated into two halves that continued to move independently, making the original single mapping irrelevant. This loss of mapping triggered a resetting process, producing a performance cost: subjects missed shape-changes when they occurred during resetting, but not when the changes occurred before or after resetting. Critically, the cost was (1) specific to the separated item, (2) larger when more mappings were lost, and (3) unaffected by the set-size. This suggests that resetting is a "local" process: VWM removes only the representations whose mappings are lost.
\end{abstract}

Electronic supplementary material The online version of this article (https://doi.org/10.3758/s13423-017-1400-y) contains supplementary material, which is available to authorized users.

Halely Balaban

halelyba@mail.tau.ac.il

1 Sagol School of Neuroscience, Tel Aviv University, $6997801 \mathrm{Tel}$ Aviv, Israel

2 School of Psychological Sciences, Tel Aviv University, 6997801 Tel Aviv, Israel

3 Psychology Department, University of Utah, Salt Lake City, UT 84112, USA
Keywords Visual working memory · Correspondence · Resetting

Visual working memory (VWM) holds task-relevant representations in an active state, ready to be manipulated by higher cognitive functions (Cowan, 2001). This active state is considered one of VWM's defining features, as manifested by the strong involvement of VWM in online processing even when stimuli remain visible, e.g., visual-search (Woodman \& Arita, 2011) and multiple object tracking (Drew \& Vogel, 2008). Furthermore, performance on change-detection tasks with and without a retention-interval is highly correlated ( $\mathrm{r} \geq 0.8$; Tsubomi, Fukuda, Watanabe, \& Vogel, 2013). These findings suggest that VWM operates both when the stimuli disappear and when they remain visible, as long as online processing is required. While most studies examined the end-points of VWM (e.g., what limits encoding or retrieval), here we focus on its online nature.

An important aspect of VWM's online status is its ability to modify its representations following the dynamic status of each item. We argue that this updating process relies on an ongoing correspondence between each representation and a specific object in the environment (Kahneman, Treisman, \& Gibbs, 1992; Levillain \& Flombaum, 2012), allowing the correct representations to be accessed and modified. While the updating mechanism is flexible enough to handle a range of changes (e.g., location: Drew \& Vogel, 2008; form: Blaser, Pylyshyn, \& Holcombe, 2000; and even interpretation: Balaban \& Luria, 2016), we suggest that certain changes cannot be integrated into existing representations. Specifically, when the object-to-representation mapping no longer corresponds to any represented item, updating is impossible, because the correct representation cannot be accessed. Instead, these circumstances trigger a resetting process, in which VWM discards existing representations and starts anew. 
To explore this process, we recently used a shape changedetection task where polygons moved during the memory array (Balaban \& Luria, 2017). This movement was task-irrelevant, and only served to manipulate objecthood. In the critical condition, a polygon moved as a coherent unit but then separated into two polygon-halves that continued to move separately. The strong common-fate Gestalt cue during the integrated movement 'convinced' VWM to treat the whole polygon as a single object (Balaban \& Luria, 2015), creating a single mapping between the polygon and a corresponding VWM-representation. When the polygon separated into two parts, this mapping became irrelevant, because VWM had to track two independent shapes, neither of which matched the original polygon. Accordingly, instead of an updating process, we found neural evidence for a resetting process: a removal of the original whole-polygon representation, followed by reencoding each polygon-half as independent representations. We monitored the contralateral delay activity (CDA; Luria, Balaban, Awh, \& Vogel, 2016; Vogel \& Machizawa, 2004) - an electrophysiological marker whose amplitude rises as more items are held in VWM. Updating, i.e., accessing the single-polygon representation and transforming it into two representations, should have produce a steady increase in the CDA amplitude, to account for the additional items (e.g., Balaban \& Luria, 2016; Drew, Horowitz, Wolfe, \& Vogel, 2012). Conversely, supporting the resetting interpretation, following the separation we found a sharp decrease in the CDA amplitude, indicating a loss of VWM-contents, followed by a rise in amplitude as VWM recovered and represented each half independently.

If this neural signature is truly indicative of resetting the contents of VWM, the process should also be associated with behavioral costs, as was found: VWM was blind to salient shape-changes that occurred during resetting, presumably because the object-to-representation mapping was lost (Balaban \& Luria, 2017). We used a novel "online change-detection" task, in which subjects indicated whether they saw an abrupt shape-change during the polygon's movement, without a retention-interval. The polygon started to move as a coherent object, and could then separate into halves that moved independently, triggering a resetting process. We manipulated the time of the shape-change: either at the moment of separation, or at two baseline intervals, $250 \mathrm{~ms}$ before or after separation. The results indicated that changes occurring simultaneously with the separation (but not before or after separation) were missed more often. Thus, without a valid mapping, accessing the correct representation is impossible, and changes at the precise moment of resetting go undetected.

Importantly, we found that this cost is specific to situations that destroy the objects-to-representations correspondence, and is not a general outcome of object-separation or a side effect of the strong perceptual cue of an item being "broken" into halves. In another experiment (Balaban \& Luria, 2017,
Experiment 7), the polygon-halves started to move independently, allowing VWM to map each half to a different representation. The halves subsequently met, moved as one polygon, and then re-separated. Thus, the separation was identical to the previous experiment, but the movement-history (and therefore the correspondence) was different, because it supported establishing a separate mapping for each polygon-half, causing the behavioral cost to diminish. While the perceptual difficulty is the same as when the two halves moved as a coherent object, it can be argued that the attentional difficulty is greater when the halves first move together, and that the reason for the larger cost in this situation is that the separation is capturing attention more when it leads to the destruction of an object. Critically, we argue that the underlying reason for the greater attentional difficulty when the polygon starts as a coherent object is that it is mapped to a single VWM representation throughout its tracking period.

Our paradigm involves a change when the items are visible, allowing us to examine VWM's sensitivity during resetting, and thus focuses on the online-processing aspect of VWM, and not on memory retention. Notably, removing the retention interval from classic change-detection tasks, with the test array immediately following the 'memory' array, does not affect behavioral performance, capacity limits, or the neural activity involved, presumably because subjects must nevertheless maintain active information (Tsubomi et al., 2013).

The current study investigated how the resetting process operates on VWM's contents. Specifically, we examined whether all VWM representations are discarded when only some of the object-to-representation mappings are lost. One option is that resetting is a "global" process that wipes out all the active representations whenever one of them is no longer mapped to an object. Alternatively, resetting might be a "local" process that removes only the representation whose mapping is lost (Ecker, Lewandowsky, \& Oberauer, 2014), leaving other VWM-contents intact.

To disentangle these hypotheses, we used the online change-detection paradigm with several moving polygons. Critically, the shape-change could happen either to a separating polygon, i.e., an item whose correspondence was lost, or to an unseparated polygon. We expected shape-changes at the time of separation to be missed when they occur to the separating polygon (replicating Balaban \& Luria, 2017). Importantly, in the current studies we focused on the outcome of the shape-change to the unseparated polygon (i.e., the polygon that kept moving as an integrated unit).

The prediction of the global-resetting hypothesis is that these changes would also be missed, since all VWMcontents are removed when one of the mappings is lost. Therefore, the intact mapping should also be discarded, preventing VWM from accessing its representation. The cost for an unseparated item might even be larger than for a separated item, because the separation involves a strong perceptual 
signal that likely draws attention to the separated item and away from the unseparated polygon. Conversely, the localresetting hypothesis predicts no cost for an unseparated item, since VWM should be able to maintain the intact mappings despite discarding the representation whose mapping was lost (i.e., separated item). However, if attention is indeed drawn away from the unseparated item, some cost will possibly be found for the unseparated item, but it should be much smaller than for the separated item.

\section{Experiment 1}

Experiment 1 investigated whether resetting operates locally or globally, by testing whether making a subset of VWM's representations inaccessible causes the other representations to be removed from VWM as well.

Additionally, we manipulated the number of separating polygons (holding set-size constant at three), such that either one or two polygons separated simultaneously. If resetting is local, losing more correspondences should produce a larger cost, since more resetting operations occur. Conversely, with a global process, the cost should be insensitive to the number of destroyed mappings, because all representations are discarded whenever one of the correspondences is lost, and thus similar costs should be found for one and two separations.

\section{Methods}

All data is available at https://osf.io/9kz7a/.

\section{Participants}

Participants were 20 (17 females, mean age 22.4 years) TelAviv University students who received partial course credit or payment for participation, and gave informed consent following the procedures of a protocol approved by the local Ethics Committee. All subjects had normal or corrected-to-normal visual-acuity and normal color-vision. Sample-size was based on Balaban \& Luria, 2017, in which the effect-size was $d=1$. Since the effect is novel, we investigated its robustness with an individual-differences analysis (see below).

\section{Stimuli and procedure}

We used an "online change-detection" task, in which changes occurred while the items were visible (Fig. 1). Each trial started with an $800 \mathrm{~ms}$ presentation of a black cross $\left(0.4^{\circ} \times\right.$ $0.4^{\circ}$ of visual angle from a viewing distance of $60 \mathrm{~cm}$ ) in the center of a gray screen, followed by the presentation of black polygons $\left(1.6^{\circ} \times 1.6^{\circ}\right)$. Each polygon was constructed by placing one of six left-half shapes next to one of six righthalf shapes (each half had a "cropped" side subtending the full length of the shape, so each pair of left- and right-halves could combine, producing 36 possible polygons). Stimuli were randomly selected for each trial, without replacement. The polygons moved in straight lines for $1400 \mathrm{~ms}$, covering $2^{\circ}$. During this movement, one of the polygon-halves could change to another polygon-half (a right half could only change to a new right-half, and a left-half could only change to a new left half), with a $50 \%$ probability. When the polygons stopped, subjects indicated whether they saw a change in one of them during their movement, via button press (on a standard computer keyboard: '/' for no change, and ' $Z$ ' for change).

Three polygons were presented on each trial. Each polygon either moved as a coherent unit throughout the trial, or separated after $800 \mathrm{~ms}$, with each half moving in a different direction. In separation trials, either one or two polygons separated, while two or one did not separate. If a change occurred in a separation trial, it was equally likely to occur in a separated or unseparated polygon.

Thus, the change trials included five conditions: three unseparated polygons ( $50 \%$ of trials), one separated item with a change in that item $(12.5 \%)$ or in one of the two unseparated items $(12.5 \%)$, and two separated polygons with a change in one of them $(12.5 \%)$ or in the unseparated polygon $(12.5 \%)$.

Regardless of the condition, changes could occur either $550 \mathrm{~ms}, 800 \mathrm{~ms}$, or $1050 \mathrm{~ms}$ after the shapes' onset, which coincides with $250 \mathrm{~ms}$ before, during, and $250 \mathrm{~ms}$ after separation, respectively.

Twelve practice trials were followed by 16 experimental blocks of 60 trials.

\section{Statistical analysis}

We analyzed hit rates using a two-way analysis of variance (ANOVA), with factors Condition and Time-of-change. We followed these tests by pairwise comparisons of conditions at time 0 (i.e., the onset of the resetting process).

\section{Results}

Our dependent measure was hit rate, meaning only trials including a change were analyzed. Importantly, it is possible to determine the identity of the changed item and the time-ofchange only for change trials (if there was no change, one cannot examine when a change occurred, and in which item). Note that accuracy rates involve both change and no-change trials. Thus, lower accuracy for the separation condition might reflect a high false-alarm rate (i.e., error-rate in no-change trials), which indicates that the separation itself was perceived as a change. However, here we were interested in whether subjects perceived the shape-change, rather than on how the separation was interpreted. For completeness, we report false alarms and reaction times in the supplementary material. 


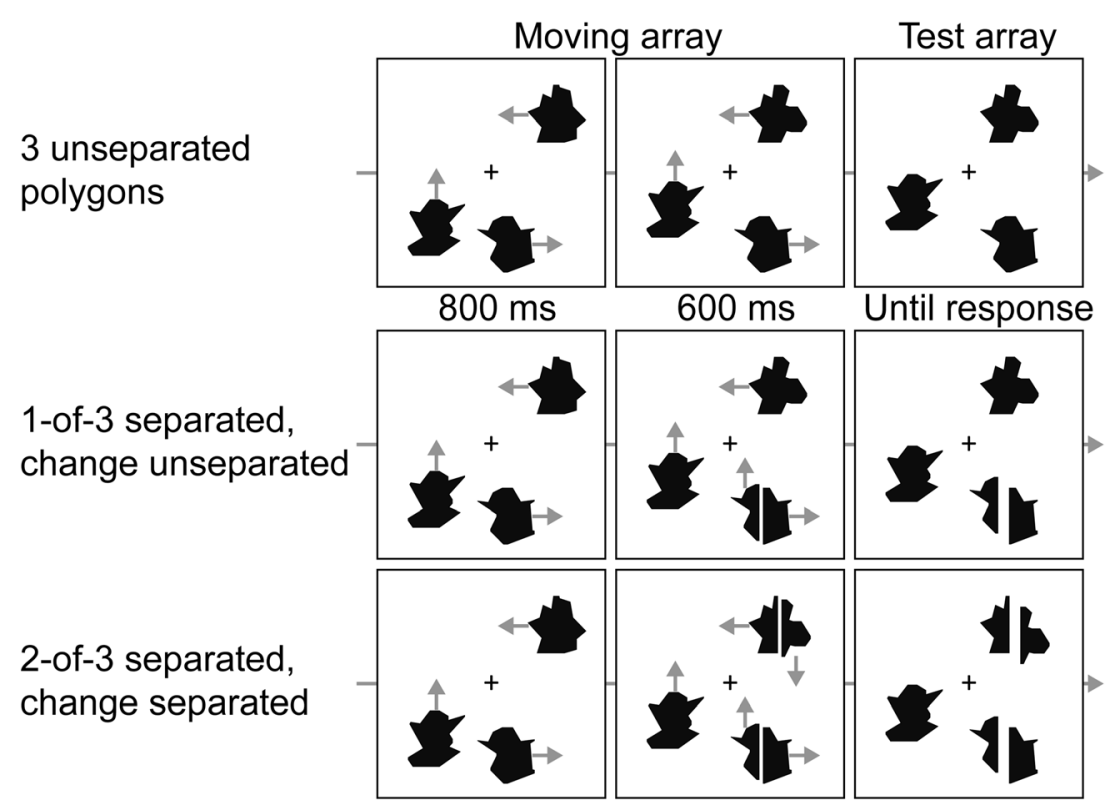

Fig. 1 The trial sequence in different conditions of Experiment 1. Three polygons moved on the screen for $1400 \mathrm{~ms}$, during which one polygon half could change (here, only change trials are presented). One or two of

Hit rates and 95\% CIs are presented in Fig. 2. There were significant effects of Condition, $F(4,76)=41.14, P<$ $.000001, \eta_{p}^{2}=.68$, and Time, $F(2,38)=48.59, P<$ $.000001, \eta_{p}^{2}=.72$, and a significant interaction, $F(8,152)=$ $44.29, P<.000001, \eta_{p}^{2}=.70$. To interpret the interaction, all analyses focused on specific comparisons at time 0 (i.e., changes coinciding with the separation). Replicating our previous findings (Balaban \& Luria, 2017), we found a cost to detect a shape-change when a polygon separated into two halves compared to integrated polygons (that did not separate). Hit rate was lower when detecting changes in both one separated polygon, $t(19)=8.04, P<.000001, \mathrm{~d}=2.52$ (Fig. 2a), and two separated polygons, $t(19)=9.28, P<.000001$, d $=2.80$ (Fig. 2b).

a

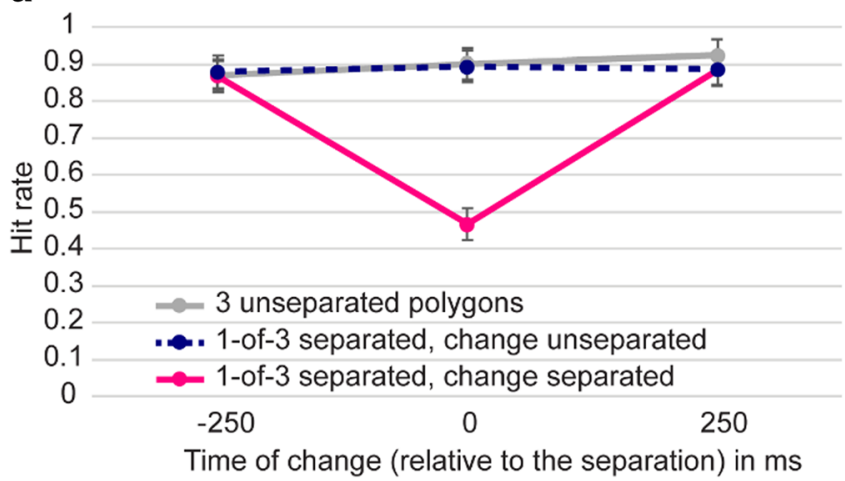

Fig. 2 a,b Hit rate in Experiment 1, by condition and time of change. Error bars depict 95\% CIs (Hollands \& Jarmasz, 2010). a One separated polygon out of three (with a change either in the separated polygon or the unseparated polygons) compared to three unseparated polygons. b Two the polygons could separate, and a change could occur in a separated item or in an unseparated item. Changes occurred in one of three intervals: during separation, or $250 \mathrm{~ms}$ before or after separation

Our main question was whether the resetting process, as exemplified by this cost, was restricted to the item whose mapping was lost, or, alternatively, whether all the representations were affected. If VWM resets all representations whenever a mapping is lost, we should observe a cost for the unseparated items as well, which should be similar in size to the separated item's cost (or even larger, given that the separation draws attention away from the unseparated items). Conversely, if VWM can maintain the still-valid mappings, there should be a minimal cost for the unseparated items, which was exactly what we found. Hit rate for changes occurring to the unseparated items during the separation of a single polygon was very high, not significantly lower than the three unseparated polygons condition, $t(19)=.37, P=.71, \mathrm{~d}=.08$.

b

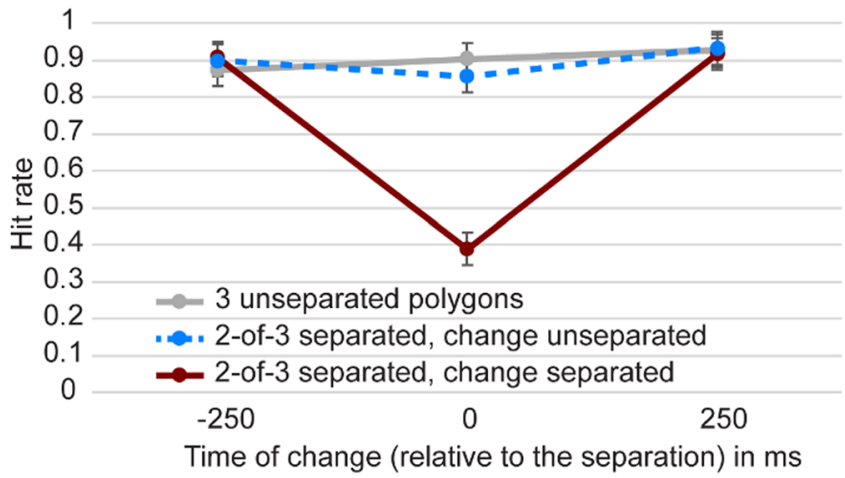

separated polygons out of three (with a change either in the separated polygons or the unseparated polygon) compared to three unseparated polygons. Note that the three unseparated polygons condition appears twice in the figure, to illustrate baseline performance 
This suggests that when one object-to-representation mapping breaks, the other mappings remain intact, allowing VWM to access these representations and update them.

When two polygons separated, there was a small but significant cost for detecting a change to the unseparated polygon: hit rate was significantly lower for changes in the unseparated polygon than for the three unseparated polygons condition, $t(19)=2.46, P<.05, \mathrm{~d}=.53$. Importantly, this effect was much smaller relative to the separated item (5\% versus $51 \%$ ). Thus, we find it unlikely that the reduction in hit rate for the unseparated item reflects a global resetting process. Presumably, attention is drawn to the separated items and away from the remaining unseparated item, resulting in this small cost. This is why this effect was evident only when two polygons separated (but not one): attention was now attracted by two events.

Another important prediction of a local process is that the cost will be larger when more correspondences are lost. Conversely, if resetting is global, such that all the representations are removed from VWM even when only one correspondence is lost, the cost should be similar regardless of the number of separating items. The results supported the local hypothesis, with a larger cost in performance for two separations, $t(19)=2.78, P<.02, \mathrm{~d}=.33$. This suggests that not all VWM's contents are removed whenever just one correspondence is lost, since breaking more correspondences produced a larger cost.

Experiment 1 established the behavioral cost produced by the resetting process: when the correspondence between a VWM representation and the object in the environment was lost, the representation could not be accessed and salient changes in the object's shape were missed. This cost was specific to the item whose correspondence was lost, while similar changes in other items were easily detected. This indicates that resetting is a local process, such that only the representations whose mappings were lost were removed from VWM. Furthermore, breaking more correspondences created a larger cost, suggesting that more resetting processes were involved, in line with the local hypothesis.

\section{Experiment 2}

The goal of Experiment 2 was to further test the local nature of resetting (supported in Experiment 1), by examining its sensitivity to the number of items maintained in VWM. In Experiment 2, only a single polygon could separate, but the set size (number of moving items) was either two or three. Hence, the number of lost mappings was held constant, while the overall number of mappings maintained in VWM was manipulated. If resetting is indeed local, the cost should be similar for a constant number of lost mappings, regardless of the overall VWM set size, because only the item whose mapping is lost becomes inaccessible.

\section{Methods}

\section{Participants}

A fresh sample of 20 Tel-Aviv University students (19 females, mean age 23.3 years) participated.

\section{Stimuli and procedure}

The stimuli and procedure were as in Experiment 1, with the following exceptions. One-half of the trials included two polygons, and the other half included three. Only a single polygon could separate. Change trials included six conditions: two unseparated polygons ( $25 \%$ of the trials), one separated polygon out of two with a change in that polygon (12.5\%) or the unseparated polygon (12.5\%), three unseparated polygons (25\%), and one separated polygon out of three with a change in that polygon $(12.5 \%)$ or one of the unseparated polygons $(12.5 \%)$.

\section{Statistical analysis}

The statistical tests were the same as in Experiment 1.

\section{Results}

Figure 3 depicts hit rates and $95 \%$ CIs. We found significant effects of Condition, $F(5,95)=19.95, P<.000001, \eta_{p}{ }^{2}=.51$, and Time, $F(2,38)=43.77, P<.000001, \eta_{p}{ }^{2}=.70$, and $\mathrm{a}$ significant interaction, $F(10,190)=28.23, P<.000001, \eta_{p}{ }^{2}=$ .60. Next, we focus of planned comparisons at time 0 that clarify these effects. First, we replicated the cost produced by resetting: a change occurring in a separating polygon was missed. Hit rate was lower when a change occurred in a separated polygon than in the integrated polygons condition, for both set size $2, t(19)=6.36, P<.000005, d=2.16$ (Fig. 3a), and 3, $t(19)=8.38, P<.000001, \mathrm{~d}=2.51$ (Fig. 3b).

Second, supporting the local nature of the resetting process, the cost was the same regardless of set-size, $t(19)=.68, P=$ $.51, \mathrm{~d}=.11$ : performance when the change occurred in a separated item at the time of separation was unaffected by the number of polygons.

Additionally, we replicated the specificity of the cost, which was limited to the item whose correspondence was lost, while similar changes in other items were easily detected. Hit rate did not significantly differ between the unseparated items and the integrated polygons, for both set size $2, t(19)=.28, P$ $=.78, \mathrm{~d}=.07$, and $3, t(19)=1.56, P=.14, \mathrm{~d}=.31$. This further supports the local nature of resetting, since only the item whose mapping was lost became inaccessible. 
a

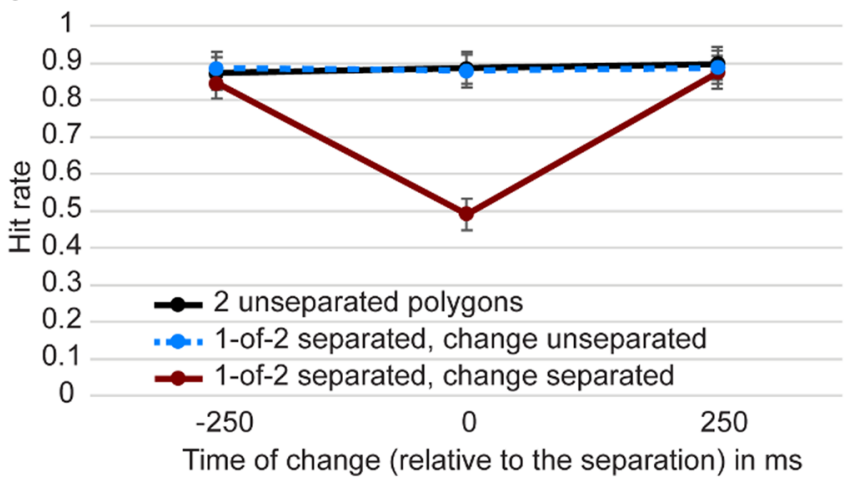

Fig. 3 Hit rate in Experiment 2, by condition and time of change. Error bars depict $95 \%$ CIs. a One separated polygon out of two (with a change either in the separated polygon or the unseparated polygon) compared to

\section{Robustness of the resetting cost}

Since VWM-resetting is a novel process, it is important to demonstrate the robustness of its behavioral marker, i.e., the cost for detecting salient changes in an item whose mapping is lost. We utilized the fact that three important conditions (three unseparated polygons, and one separated polygon out of three with a change in the separated item or the unseparated items) were common to both experiments, and examined hit rate for each subject individually (Fig. 4).

The behavioral cost was found for each one of our 40 subjects: hit rate at time 0 was lower for a separated polygon than for three unseparated items, suggesting the effect is highly robust. Furthermore, the local nature of resetting was also supported in each one of our 40 subjects, with the hit rate being lower for the separated than the b

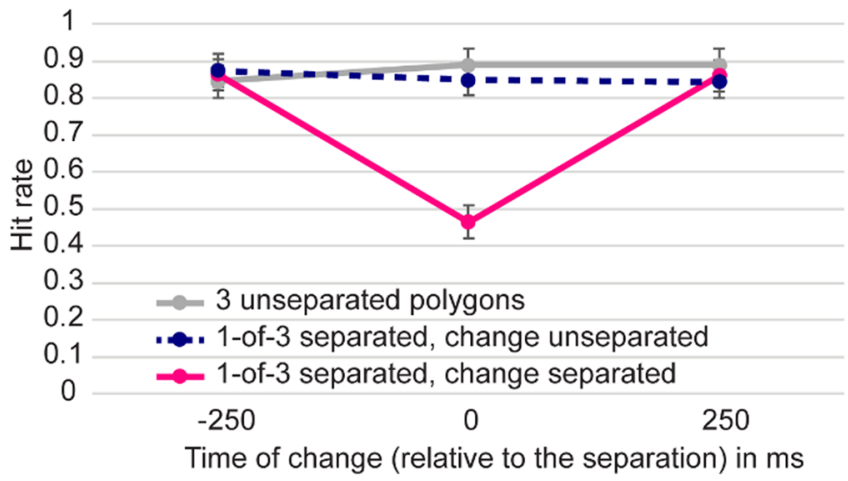

two unseparated polygons. b One separated polygon out of three (with a change either in the separated polygon or the unseparated polygons) compared to three unseparated polygons

unseparated polygon. Note that some subjects' hit rate is very low, indicating they responded "same" more than "different" on separation trials including changes. For example, a participant with a $20 \%$ hit rate missed $80 \%$ of the trials that actually included a change, due to the separation.

Notably, hit rate might actually be an inflated measure of performance, because the separation itself is a salient perceptual event, which could have been perceived as a sort of change (for further evidence, see the Supplementary Material). Importantly, if participants interpreted the object-separation as a change, this would translate to a correct response on trials that also included a shape-change, thus inflating hit rate. Thus, interpreting the separation as a change cannot account for the low hit rate we observed for several subjects.

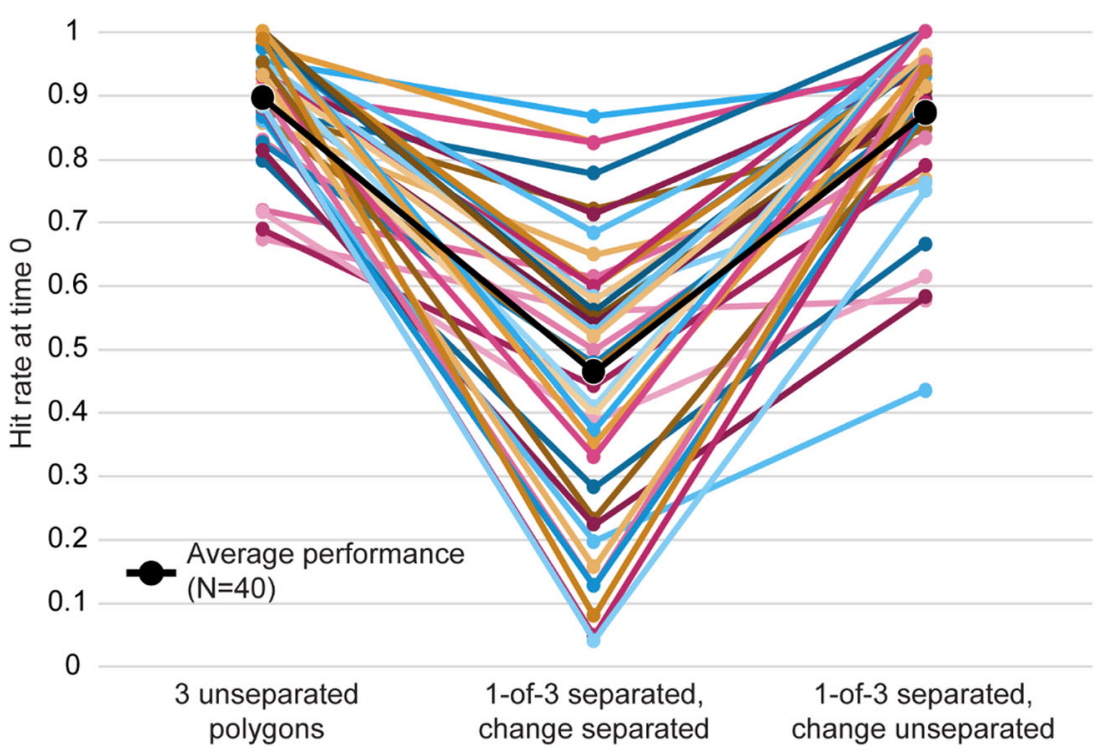

Fig. 4 Individual performance in Experiments 1 and 2. We present hit rates at time 0 for each one of our subjects, in the three conditions that were included in both experiments: three integrated polygons (left), and one separated polygon out of three, with a change either in that item (middle) or in an unseparated item (right). Black line depicts average performance 


\section{Discussion}

We tested whether VWM-resetting operates "locally", on an item-specific basis, or "globally", removing all VWMcontents whenever one of the objects-to-representations mapping is lost. Both experiments supported the local hypothesis. While changes during resetting were missed for an item whose mapping was lost, similar changes in other items were accurately detected, suggesting that their mappings remained accessible. Furthermore, the resetting cost was larger when more correspondences were lost (Experiment 1), and was unaffected by the overall number of representations (i.e., set-size; Experiment 2). This shows that VWM selectively removes only the items that are no longer mapped to an object in the environment, with more resetting operations producing a larger performance cost. Additionally, this demonstrates that two mappings cannot be removed in parallel, which would have produced a similar cost regardless of the number of separating items. This provides further support for the local nature of resetting, which apparently can only operate on a single item at a time. Our results suggest that resetting is a highly efficient process that does not lead to a costly wipeout of all VWM's contents. Instead, the representations whose mappings are still intact remain accessible, while the inaccessible representations are selectively discarded.

The present results replicated and established the large cost in performance for the item whose correspondence was lost (Balaban \& Luria, 2017): hit rate was very low when changes occurred simultaneously with separation, i.e., with the onset of the resetting process. This is not due to the perceptual signal of the object separation per se, since similar changes in other items (which should have received less attention) were easily detected. Notably, the cost was found for every one of our participants (Fig. 4), suggesting that it is very robust. Importantly, we argue that the triggering factor of resetting is the loss of object-to-representation correspondence, and that updating is only possible if these mappings exist.

The notion of a correspondence relates to the object file theory (Kahneman et al., 1992), which suggested a pointerlike system connecting representations and objects. In this framework, objects in the environment are linked via a correspondence process to temporary object representations that include their different features. Additionally, object files are assumed to be defined and accessed by location, in line with our finding that separating a shape into independently moving halves triggers resetting (however, note that we previously found resetting also in object-switching, wherein a shape was replaced by a different shape in the same location; Balaban \& Luria, 2017). Thus, our results of a behavioral cost associated with correspondence-loss situations provide strong support for the theoretical claims of the object file framework.

Since VWM is highly capacity-limited, a process for removing no-longer-relevant information is necessary for
VWM to stably represent the dynamic environment. Resetting could thus be involved, for example, in learning and reading, where obsolete schemas must be discarded as new information is accumulated. The evidence we found for the local nature of the resetting process suggest it can effectively operate in complex situations, where some of the representations are still relevant and some are not. More research is necessary to determine whether the resetting process we have uncovered is a strategy, or a more bottom-up process.

Acknowledgments This work was supported by a Binational Science Foundation (BSF) grant number 2015301 awarded to R.L. and T.D. H.B. was supported by an Azrieli Fellowship. The authors declare no competing financial interests.

\section{References}

Balaban, H., \& Luria, R. (2015). The number of objects determines visual working memory capacity allocation for complex items. NeuroImage, 119, 54-62.

Balaban, H., \& Luria, R. (2016). Object representations in visual working memory change according to the task context. Cortex, 81, 1-13.

Balaban, H., \& Luria, R. (2017). Neural and behavioral evidence for an online resetting process in visual working memory. The Journal of Neuroscience, 37(5), 1225-1239.

Blaser, E., Pylyshyn, Z. W., \& Holcombe, A. O. (2000). Tracking an object through feature space. Nature, 408(6809), 196-199.

Cowan, N. (2001). The magical number 4 in short-term memory: A reconsideration of mental storage capacity. The Behavioral and Brain Sciences, 24(1), 87-114; discussion 114-185.

Drew, T., Horowitz, T. S., Wolfe, J. M., \& Vogel, E. K. (2012). Neural measures of dynamic changes in attentive tracking load. Journal of Cognitive Neuroscience, 24(2), 440-450.

Drew, T., \& Vogel, E. K. (2008). Neural measures of individual differences in selecting and tracking multiple moving objects. The Journal of Neuroscience, 28(16), 4183-4191.

Ecker, U. K., Lewandowsky, S., \& Oberauer, K. (2014). Removal of information from working memory: A specific updating process. Journal of Memory and Language, 74, 77-90.

Hollands, J. G., \& Jarmasz, J. (2010). Revisiting confidence intervals for repeated measures designs. Psychonomic Bulletin \& Review, 17(1), $135-138$

Kahneman, D., Treisman, A., \& Gibbs, B. J. (1992). The reviewing of object files: Object-specific integration of information. Cognitive Psychology, 24(2), 175-219.

Levillain, F., \& Flombaum, J. I. (2012). Correspondence problems cause repositioning costs in visual working memory. Visual Cognition, 20(6), 669-695.

Luria, R., Balaban, H., Awh, E., \& Vogel, E. K. (2016). The contralateral delay activity as a neural measure of visual working memory. Neuroscience \& Biobehavioral Reviews, 62, 100-108.

Tsubomi, H., Fukuda, K., Watanabe, K., \& Vogel, E. K. (2013). Neural limits to representing objects still within view. The Journal of Neuroscience, 33(19), 8257-8263.

Vogel, E. K., \& Machizawa, M. G. (2004). Neural activity predicts individual differences in visual working memory capacity. Nature, 428(6984), 748-751.

Woodman, G. F., \& Arita, J. T. (2011). Direct electrophysiological measurement of attentional templates in visual working memory. Psychological Science, 22(2), 212-215. 